Dr. Rolf Malessa, Weimar. Verminderte Lebensfreude, Schlafstörungen und eingeschränkte Aktivität sind die Folge.

\section{Frühzeitig intervenieren}

Damit sich die funktionellen Beeinträchtigungen und die Schmerzempfindung nicht gegenseitig aufschaukeln, muss zeitnah interveniert werden. Gute Erfahrungen hat der Schmerzexperte Malessa mit dem selektiven Serotonin- und Noradrenalin-Wiederaufnahmehemmer (SSNRI) Duloxetin (Ariclaim ${ }^{\circledR}, 60$ oder 120 $\mathrm{mg} / \mathrm{d}$ ) gemacht. Die gute analgetische Wirksamkeit der Substanz bei schmerzhafter DPNP beruht auf einer Stärkung der endogenen Schmerzhemmung über die deszendierenden inhibitorischen Schmerzbahnen des ZNS.
Schon drei Tage nach Therapiebeginn zeigte sich in klinischen Studien ein gegenüber Placebo signifikanter Effekt. Nach sieben Tagen erreichten 40\% der Patienten eine Schmerzreduktion um mindestens 30\%. „Eine 30\%ige Schmerzlinderung bedeutet eine klinisch relevante Verbesserung“, erläuterte Malessa. Durch die Reduktion der Schmerzen bessern sich auch die schmerzbedingten funktionellen Beschwerden. Die ausgeprägte analgetische Wirksamkeit des SSNRI bei der schmerzhaften DPNP und seine gute Verträglichkeit können somit den Patienten wieder einen aktiven Alltag ermöglichen.

- Abdol A. Ameri

Pressegespräch, Berlin, 8. Oktober 2009

(Veranstalter: Boehringer Ingelheim und Lilly)

\section{Kurz notiert}

Neues Medikament gegen Vorhofflimmern - Die europäische Kommission hat dem Antiarrhythmikum Dronedaron (Multaq ${ }^{\circledR}$ ) die Zulassung erteilt. Dronedaron ist indiziert bei erwachsenen, klinisch stabilen Patienten mit nicht permanentem Vorhofflimmern (VHF), um das Wiederauftreten von VHF zu verhindern oder die ventrikuläre Herzfrequenz zu senken. In der ATHENA-Studie reduzierte das Antiarrhythmikum bei VHF-Patienten das Risiko für kardiovaskulär bedingte Krankenhausaufenthalte oder Tod jeglicher Ursache um relativ $24 \%$. Sanofi-Aventis

\title{
Neues COPD-Medikament
}

\section{Schnelle Bronchodilatation, die 24 Stunden anhält}

- Zur Behandlung der COPD gibt es jetzt einen neuen lang wirksamen Beta-2-Agonisten (LABA). Indacaterol ist das erste in Europa zugelassene COPD-Medikament, das bei einmal täglicher Inhalation für eine Bronchodilatation über 24 Stunden sorgt und dessen Wirkung bereits innerhalb weniger Minuten eintritt.

Es handelt sich um eine neue Substanzklasse, die sich auch strukturell von den bisher verfügbaren LABA Salmeterol und Formoterol unterscheidet, erläuterte Dr. Alexandre Trifilieff vom Unternehmen Novartis. Indacaterol habe die gleiche Lipophilie wie Salmeterol, interagiere aber stärker mit der RegioPn der Beta-2-Rezeptoren, was die 24-Stunden-Wirkung bedinge. Der schnelle Wirkeintritt im Vergleich zu Salmeterol sei einer höheren intrinsischen Effektivität zu verdanken, so der Wissenschaftler. Die für die Relaxation der glatten Gefäßmuskulatur notwendige CAMP-Konzentration werde rascher erreicht.

\section{Vergleich mit anderen Bronchodilatatoren}

Im klinischen Studienprogramm hat sich die gute Wirksamkeit und Verträglichkeit von Indacaterol (Onbrez ${ }^{\circledR}$ Breezhaler $^{\circledR}$ ) bestätigt, berichtete Prof. Dr. Gerhard König, Memmingen. Die FEV 1 am Ende des Dosisintervalls war im Vergleich zu Placebo um $180 \mathrm{ml}$ und im Vergleich zu Formoterol und Salmeterol um 50-110 ml größer. Im Vergleich zu Tiotropium (18 $\mu \mathrm{g} 1 \mathrm{x}$ tgl.) ergab sich bei beiden zugelassenen Indacateroldosierungen (150 und $300 \mu \mathrm{g}$ $1 \times$ tgl.) ebenfalls im Trend eine bessere Wirkung auf die Lungenfunktion. Die

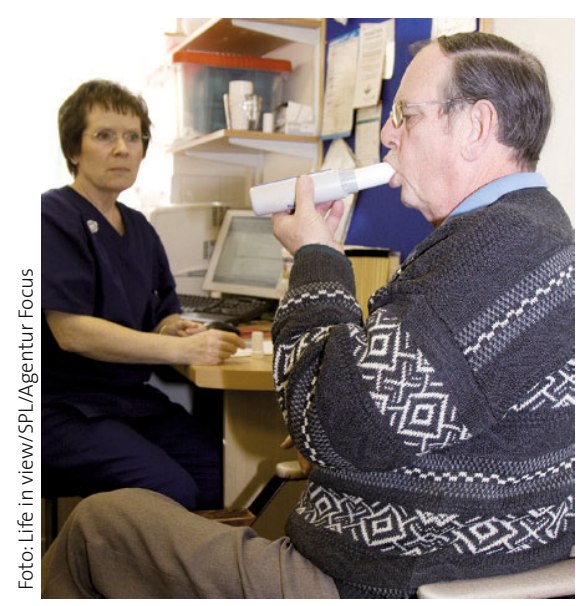

Mit Indacaterol ist die $\mathrm{FEV}_{1}$ am Ende des Dosisintervalls größer als mit den bisher verfügarem LABA.
Besserung blieb über die gesamte Studiendauer von 26 Wochen stabil, so König. Die Bedarfsmedikation konnte unter der Therapie reduziert werden. Nach einem halben Jahr kam die Hälfte der Patienten ganz ohne Bedarfsmedikamente aus.

Derzeit liegen Erfahrungen über 52 Wochen vor, die die bisherigen Ergebnisse bestätigen. Auch die Lebensqualität der COPD-Patienten wurde im Trend gebessert, erläuterte König. Bei etwa jedem zweiten Patienten überschritt die Besserung das klinische Signifikanzniveau von vier Score-Punkten. Die Therapie wurde gut vertragen, Hauptnebenwirkung ist Husten direkt nach der Applikation. Systemische Effekte gab es in den Studien quasi nicht, berichtete König. Die Herzfrequenz erhöhte sich im Mittel um weniger als einen Schlag pro Minute, Tachykardien, auffällige OTcF-Intervalle oder Hypokaliämien waren auf Placeboniveau.

Derzeit wird Indacaterol im Studienprogramm INERGIZE-COPD bei fast 6000 Patienten eingehender untersucht.

\footnotetext{
- Sonja Böhm

Quelle: Einführungspressekonferenz Onbrez ${ }^{\circledR}$ Frankfurt/M., 18. November 2009 (Novartis Pharma $\mathrm{GmbH}$ )
} 